\title{
Nonlinear Generalization of Singular Vectors: Behavior in a Baroclinic Unstable Flow
}

\author{
OLIVIER RiVIÈRE \\ Laboratoire de Météorologie Dynamique/IPSL, Ecole Normale Supérieure/CNRS, Paris, and Ecole Nationale des Ponts et Chaussées, \\ Marne la Vallée, France \\ Guillaume Lapeyre And Olivier TAlagrand \\ Laboratoire de Météorologie Dynamique/IPSL, Ecole Normale Supérieure/CNRS, Paris, France
}

(Manuscript received 21 December 2006, in final form 28 September 2007)

\begin{abstract}
Singular vector (SV) analysis has proved to be helpful in understanding the linear instability properties of various types of flows. SVs are the perturbations with the largest amplification rate over a given time interval when linearizing the equations of a model along a particular solution. However, the linear approximation necessary to derive SVs has strong limitations and does not take into account several mechanisms present during the nonlinear development (such as wave-mean flow interactions). A new technique has been recently proposed that allows the generalization of SVs in terms of optimal perturbations with the largest amplification rate in the fully nonlinear regime. In the context of a two-layer quasigeostrophic model of baroclinic instability, the effect of nonlinearities on these nonlinear optimal perturbations [herein, nonlinear singular vectors (NLSVs)] is examined in terms of structure and dynamics. NLSVs essentially differ from SVs in the presence of a positive zonal-mean shear at initial time and in a broader meridional extension. As a result, NLSVs sustain a significant amplification in the nonlinear model while SVs exhibit a reduction of amplification in the nonlinear model. The presence of an initial zonal-mean shear in the NLSV increases the initial extraction of energy from the total shear (basic plus zonal-mean flows) and opposes wave-mean flow interactions that decrease the shear through the nonlinear evolution. The spatial shape of the NLSVs (and especially their meridional elongation) allows them to limit wave-wave interactions. These wave-wave interactions are responsible for the formation of vortices and for a smaller extraction of energy from the basic flow. Therefore, NLSVs are able to modify their shape in order to evolve quasilinearly to preserve a large nonlinear growth. Results are generalized for different norms and optimization times. When the streamfunction variance norm is used, the NLSV technique fails to converge because this norm selects very small scales at initial time. This indicates that this technique may be inadequate for problems for which the length scale of instability is not properly defined. For other norms (such as the potential enstrophy norm) and for different optimization times, the mechanisms of the NLSV amplification can still be viewed through wave-wave and wave-mean flow interactions.
\end{abstract}

\section{Introduction}

The properties of baroclinic instability have been extensively studied in linear approximation since the pioneering work of Charney (1947) and Eady (1949). The crucial characteristics of the mean state for the development of the instability [in terms of potential vorticity (PV) gradients] have been identified (Charney and Stern 1962; Bretherton 1966; Pedlosky 1987). Two tech-

Corresponding author address: G. Lapeyre, Laboratoire de Météorologie Dynamique, Ecole Normale Supérieure, 24 Rue Lhomond, 75005 Paris, France.

E-mail: glapeyre@lmd.ens.fr niques are traditionally used to study the linear instability problem. The first one uses the normal mode (NM) approach, that is, linearizing a model about a mean state and finding a solution asymptotically growing in time. Such a method presents the disadvantage that it fails to capture localized disturbances that can have a rapid growth over a limited period in time (Farrell 1982). A second approach consists of identifying "optimal perturbations" [called singular vectors (SVs)] that maximize the growth rate over a given time interval (Farrell 1982; Lacarra and Talagrand 1988; Farrell and Ioannou 1996), thus permitting diagnosis of regions in the physical space where small disturbances can have an explosive growth. This idea has been applied in pre- 
dictability studies, and it has been shown that SVs capture the essential ingredients of growth of extratropical synoptic systems (Badger and Hoskins 2001; Buizza and Palmer 1995; Hoskins et al. 2000, among others). However, their main disadvantage is that they are based on a linearization of the equations of motion. Under the linear assumption, positive and negative perturbations have the same growth rate although they can evolve rather differently under nonlinear dynamics (Gilmour et al. 2001; Reynolds and Rosmond 2003; Hoskins and Coutinho 2005). In addition, the growth of the singular vectors in the nonlinear system can be greatly reduced compared to the growth in the linear case. We may then wonder if the optimality of singular vectors is still valid for the original nonlinear problem.

Recently, Mu (2000) has developed a new method to extend the concept of optimal perturbations to the nonlinear regime. The idea is to find a perturbation of a given model solution that will be an extremum in terms of the amplification rate of the perturbation energy over a finite time interval. This solution will be referred to as the nonlinear singular vector (NLSV) throughout the paper. The energy of the perturbation is constrained to a fixed value at initial time. A solution of such a problem can be computed using numerical techniques available for large-scale nonlinear optimization problems. A second and similar technique called conditional nonlinear optimal perturbations (CNOPs) was developed by $\mathrm{Mu}$ et al. (2003). CNOPs are the perturbations with the largest final energy for an initial energy smaller than a given value. This technique has been applied in a simple ENSO model (Duan et al. 2004), in a two-box model of the thermohaline circulation (Mu et al. 2004), and also in a problem of equivalent barotropic instability (Mu and Zhang 2006). One can also mention the work of Barkmeijer (1996), who has developed an iterative method for the same purpose.

Even if it is generally admitted that the linear approximation is valid for up to about two days for largescale meteorology, optimized perturbations for the nonlinear problem are important in situations of instability of well-formed coherent structures (Snyder 1999) or when strongly nonlinear and intermittent processes are at play (e.g., in the case of latent heat release by large-scale or convective precipitation). This is one motivation for this work. To understand the potentials of this new technique, we have chosen to apply it to the baroclinic instability problem in a quasigeostrophic (QG) two-layer model. The Phillips (1954) model is a good test case to study the baroclinic wave development because its properties in terms of linear and nonlinear dynamics are well known.
The paper is organized as follows: in the next section, we succinctly describe the QG model we use. Then we discuss the algorithm to compute the leading singular vector and the nonlinear optimal perturbations. In section 4, we compare the properties of the leading SV and NLSVs in terms of spatial evolution and amplification rate. Then, in section 5, we present two mechanisms to explain how NLSVs are able to adjust in order to maximize their growth rate and limit nonlinearities. These mechanisms are related to wave-mean flow and wavewave interactions. Section 6 examines the impact of using different norms and optimization times. Finally, conclusions are drawn.

\section{Numerical model}

The QG potential vorticity equations in the two-layer model on the $\beta$ plane can be written as

$$
\begin{aligned}
& \partial_{t} q_{1}+J\left(\psi_{1}, q_{1}\right)+\beta \partial_{x} \psi_{1}=0 \quad \text { and } \\
& \partial_{t} q_{2}+J\left(\psi_{2}, q_{2}\right)+\beta \partial_{x} \psi_{2}=0,
\end{aligned}
$$

where

$$
\begin{aligned}
& q_{1}=\nabla^{2} \psi_{1}+\lambda^{-2}\left(\psi_{2}-\psi_{1}\right) \text { and } \\
& q_{2}=\nabla^{2} \psi_{2}+\lambda^{-2}\left(\psi_{1}-\psi_{2}\right)
\end{aligned}
$$

are the upper- and lower-layer potential vorticities, $\psi_{i}(i=1,2)$ the associated streamfunctions, and $\lambda$ the Rossby deformation radius. Here, $J(A, B)=\partial_{x} A \partial_{y} B-$ $\partial_{y} A \partial_{x} B$. All the equations that will be presented here are nondimensionalized following Pedlosky (1987) and Rivière et al. (2001). We first decompose the potential vorticity $q$ into a perturbation $\hat{q}$ and a stationary basic state $Q$. The equations for PV perturbations are

$$
\partial_{t} \hat{q}_{1}+J\left(\hat{\psi}_{1}, \hat{q}_{1}\right)+J\left(\Psi_{1}, \hat{q}_{1}\right)+J\left(\hat{\psi}_{1}, Q_{1}\right)+\beta \partial_{x} \hat{\psi}_{1}=0
$$

and

$$
\partial_{t} \hat{q}_{2}+J\left(\hat{\psi}_{2}, \hat{q}_{2}\right)+J\left(\Psi_{2}, \hat{q}_{2}\right)+J\left(\hat{\psi}_{2}, Q_{2}\right)+\beta \partial_{x} \hat{\psi}_{2}=0,
$$

where $\Psi_{1}$ and $\Psi_{2}$ are the basic-state streamfunctions of the upper and lower layers, and $\hat{\psi}_{1}$ and $\hat{\psi}_{2}$ are their analogs for the perturbations. The linearization of these equations yields

$$
\begin{aligned}
& \partial_{t} \hat{q}_{1}+J\left(\Psi_{1}, \hat{q}_{1}\right)+J\left(\hat{\psi}_{1}, Q_{1}\right)+\beta \partial_{x} \hat{\psi}_{1}=0 \quad \text { and } \\
& \partial_{t} \hat{q}_{2}+J\left(\Psi_{2}, \hat{q}_{2}\right)+J\left(\hat{\psi}_{2}, Q_{2}\right)+\beta \partial_{x} \hat{\psi}_{2}=0 .
\end{aligned}
$$


The hats will be omitted in the following discussion. We choose a basic state that is zonally symmetric with zonal velocity equal to

$$
U_{1}(y)=-U_{2}(y)=\frac{U}{2 \cosh ^{2}(y / L)},
$$

where $U_{i}=-\partial_{y} \Psi_{i}(i=1,2)$. (The meridional profile of the shear of the basic flow is presented in Fig. 6a.) The parameters used in this study are those of Rivière et al. (2001) who studied the structure of SVs and normal modes for a similar model. For a typical velocity scale $U=7.35 \mathrm{~m} \mathrm{~s}^{-1}$, a deformation radius $\lambda=507 \mathrm{~km}$, a meridional domain size of $3744 \mathrm{~km}$, a Brunt-Väisälä frequency of $1.13 \times 10^{-2} \mathrm{~s}^{-1}, f_{0}=10^{-4} \mathrm{~s}^{-1}$, and a jet scale of $L=600 \mathrm{~km}$, we introduce nondimensionalized variables (with "adim" subscripts) $U_{\text {adim }}=2, \lambda_{\text {adim }}^{-2}=$ $54.53, \beta_{\text {adim }}=32.4$, and $L_{\text {adim }}=1$. The basic state we use here is a stationary and baroclinically unstable solution of (3a) and (3b) with a change in sign of the PV gradient between the two layers. Moreover, it is barotropically stable because the PV gradient has a constant sign in each layer. The spatial resolution is $256^{2}$. The numerical model is pseudospectral over a doubly periodic domain with size $[2 \pi, 2 \pi]$. The code was initially developed by Smith and Vallis (2002).

\section{Numerical computation of leading $S V$ and NLSV}

The leading SV is the solution of the linearized Eqs. (4a) and (4b) with the largest amplification rate for a given norm over a finite time. We first use the total energy norm for our optimization problem. Total energy is defined as the sum of the kinetic and potential energies,

$$
E(t)=\frac{1}{2}\left\langle\left|\nabla \psi_{1}\right|^{2}+\left|\nabla \psi_{2}\right|^{2}+2 \lambda^{-2}\left(\psi_{1}-\psi_{2}\right)^{2}\right\rangle,
$$

where \langle\rangle denotes the spatial horizontal mean. The leading SV can be viewed as the eigenvector associated with the largest eigenvalue of the matrix (in spectral space variables) involved in the Rayleigh quotient $E(t=$ $\left.T_{\text {opt }}\right) / E(t=0)$. Here $T_{\text {opt }}$ is the optimization time. A Lanczos method can then be used to compute the SV with the help of the adjoint equations of (4a) and (4b); see Buizza and Palmer (1995) for a complete description of the procedure.

The NLSV is a maximum (possibly local; see below) of the scalar function

$$
f(q)=\frac{E\left(t=T_{\mathrm{opt}}\right)}{E(t=0)}
$$

under the constraint $c(q) \equiv E(t=0)-E_{0}=0$. Here $E_{0}$ is the initial energy of the perturbation and $E(t)$ is the total energy after the evolution in the nonlinear model [(3a), (3b)]. The variable $q$ represents the initial state $q_{1}, q_{2}$. This is a standard problem in large-scale constrained nonlinear optimization, which we solve here with a quasi-Newton Broyden-Fletcher-GoldfarbShanno (BFGS) algorithm called IPOPT (Wächter and Biegler 2006). The algorithm proceeds in minimizing the inverse of the cost function, that is, $1 / f(q)$. It needs to evaluate the gradients of the function $f(q)$ and the constraint $c(q)$, which are computed using the adjoint equations of the model. The algorithm also approximates the Hessian by the BFGS method. Further details can be found in Wächter and Biegler (2006). The algorithm stops when the optimality error $\epsilon_{\text {err }}$, computed as

$$
\boldsymbol{\epsilon}_{\mathrm{err}}=\max \left\{\|\boldsymbol{\nabla}[1 / f(q)]+\mu \boldsymbol{\nabla} c(q)\|_{\infty}, \alpha\|c(q)\|_{\infty}\right\},
$$

where $\alpha$ is a scaling factor and $\mu$ the Lagrangian multiplier for the constraints that are implicitly defined by the numerical algorithm and are of order unity, becomes less than a specified error tolerance $\epsilon$. We have found that the rate of convergence of the technique depends on the spectral resolution. If two wavenumbers are associated with almost equal growth rates, then the algorithm may not converge toward either one and tends instead to wander from one mode to the other. This may be the case if there is not enough spatial resolution, or if the parameter $\epsilon$ is not small enough. To reduce this tendency, the algorithm is stopped in most cases after 50 iterations. In practice, it takes less than 20 iterations for the algorithm to converge for $\epsilon=10^{-4}$ (except for $E_{0} \geq 1$ ).

One would like a method to find an NLSV that is known to be a global maximum for the amplification rate. However, our numerical method cannot ensure this. In practice, we find an NLSV that is close to the global maximum amplification by initializing the NLSV algorithm starting from the leading SV or its opposite as a first guess (in the nonlinear model they can have a different amplification). For small enough perturbations, the NLSV and the leading SV will be almost the same. In this way, for small enough energy $E_{0}$, the solution should be close to the global maximum.

\section{Comparison of normal mode, leading SV, and NLSVs}

First, we describe the properties of the leading singular vector and normal mode. The normal mode was computed through a linear integration of the model with a time sufficiently long that the exponential 

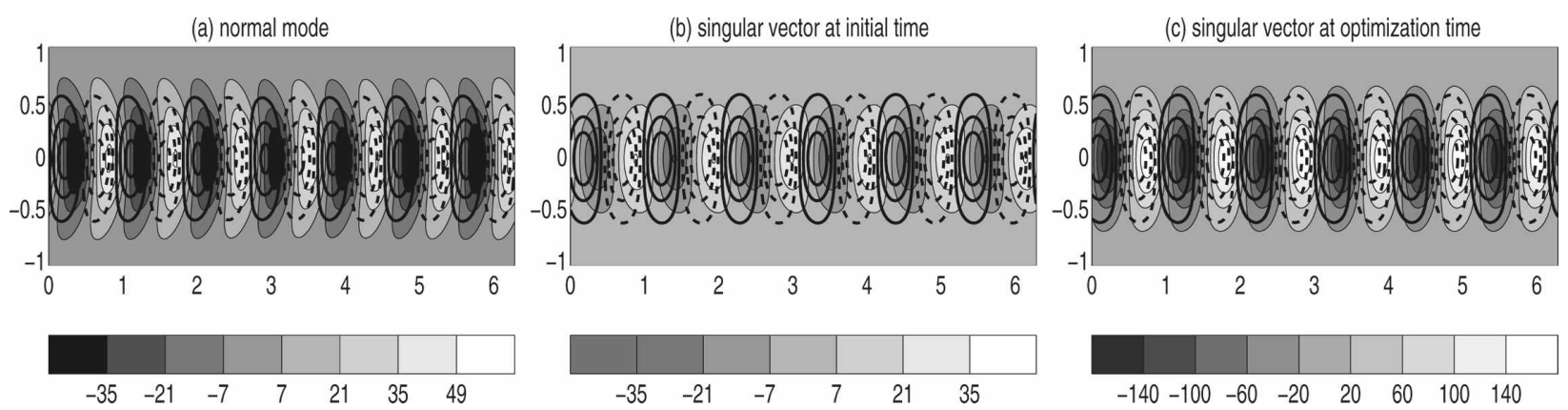

FIG. 1. Snapshot of (a) normal mode with an initial energy $E_{0}=0.5$, the leading SV (b) at initial time and (c) at $t=T_{\text {opt }}$ (in the linear tangent model). The filled contours represent the upper-layer potential vorticity and the solid and dashed contours represent the lower-layer PV (solid for positive values and dashed for negative values). Negative and positive values have the same contour intervals.

growth rate becomes almost constant. For the SV and NLSV, we have chosen an optimization time $T_{\text {opt }}=0.3$ for which the linear and nonlinear evolutions of the SV with initial energy of $E_{0}=0.5$ significantly differ. We will discuss the impact of different optimization times and different choices of norms in section 6 . In dimensional units, $T_{\mathrm{opt}}=0.3$ corresponds to an integration of $42 \mathrm{~h}$. The leading SV and NM have respective linear amplification rates in total energy $E\left(t=T_{\text {opt }}\right) / E(t=0)$ of 9.85 and 8.38. They are concentrated along the axis of the basic jet (see Figs. 1a,b) and have a zonal wavenumber equal to 7 for the NM and 6 for the SV. We will see in section 6 that for a longer optimization time, the dominant zonal wavenumber and structure of the leading SV converges toward those of the NM. During the time evolution, the SV moves eastward and the upperand lower-layer waves mutually amplify (cf. Figs. 1b,c). The upper and lower PV anomalies of the leading SV are approximately in phase quadrature at initial time and close to phase opposition at final time. The upper and lower PV anomalies of the normal mode are also in phase opposition. This means that the temperature signal $\left(\psi_{1}-\psi_{2}\right)$ dominates in the PV signal [see Eqs. (2a), (2b)].

\section{a. SV evolution}

If we let the SV evolve in the nonlinear model for an initial energy of $E_{0}=0.5$, we observe that the amplification is much smaller (6.86; see Table 1). A similar result is obtained for the normal mode (which has nonlinear amplification 4.99 for $E_{0}=0.5$ ). We will not discuss the nonlinear evolution of the NM in the following because it is qualitatively similar to the SV except for the reduced amplification. To understand the behavior of the leading SV, one can look at the SV at the optimization time (Fig. 2). For small initial energies (Fig. 2a), the SV at final time resembles the SV in the linear model (cf. Fig. 1c). When the initial energy in- creases (Figs. 2b,c), we see that PV anomalies tend to move strongly in the meridional direction, whereas their displacement in the zonal direction is similar to the linear case. Upper-layer positive PV anomalies and lower-layer negative anomalies move toward the equator, whereas upper-layer negative PV anomalies and lower-layer positive anomalies move toward the pole. This movement contributes to a meridional PV flux corresponding to a net poleward transport of heat that is typical of the development of baroclinic waves (Pedlosky 1987; Heifetz et al. 2004). For values of initial energy of the order of 0.5 and larger, we see the development of vortices (Fig. 2d). Both phenomena (meridional displacement and formation of vortices) are related to wave-wave and wave-mean flow interactions as we will see later. This has profound consequences for the amplification of the SV in the nonlinear model because it decays by a factor of 3 when the initial energy is increased to $E_{0}=5$ (solid curve in Fig. 3). Indeed, the $\mathrm{SV}$ amplification rate decreases very rapidly when increasing initial energy beyond $E_{0}>5 \times 10^{-2}$. A similar result was obtained by Snyder and Joly (1998) for a growing baroclinic wave in the Eady model. It is possible here to predict the energy for which nonlinear terms will become important. Such a situation will occur when linear and nonlinear terms balance each other in (3a) and (3b), that is, when $\partial_{t} q_{i} \approx J\left(\psi_{i}, q_{i}\right)$. If we approximate $q_{i}(t)$ by $q_{i}(t=0) \exp (\lambda t)$, we have $\partial_{t} q_{i} \approx$ $\lambda q_{i}(t)$. The advection term $J\left(\psi_{i}, q_{i}\right)$ can be scaled as

TABLE 1. Amplification rates of energy for NM, SV, and NLSV in different models: L, linear Eqs. (4a), (4b); NL, nonlinear Eqs. (3a), (3b); and WKNL, weakly nonlinear Eqs. (9a), (9b). In each case $E_{0}=0.5$.

\begin{tabular}{|c|c|c|c|c|c|c|}
\hline Structure & $\begin{array}{l}\mathrm{NM} \\
\text { in } \mathrm{L}\end{array}$ & $\begin{array}{l}\text { SV } \\
\text { in } \mathrm{L}\end{array}$ & $\begin{array}{c}\text { SV } \\
\text { in NL }\end{array}$ & $\begin{array}{c}\mathrm{SV} \text { in } \\
\mathrm{WKNL}\end{array}$ & NLSV & $\begin{array}{c}\text { NLSV in } \\
\text { WKNL }\end{array}$ \\
\hline Amplification & 8.38 & 9.85 & 6.86 & 6.71 & 8.21 & 8.13 \\
\hline
\end{tabular}


(a) SV with $E_{-} 0=5 \times 10^{\wedge}-4$ at optimization time

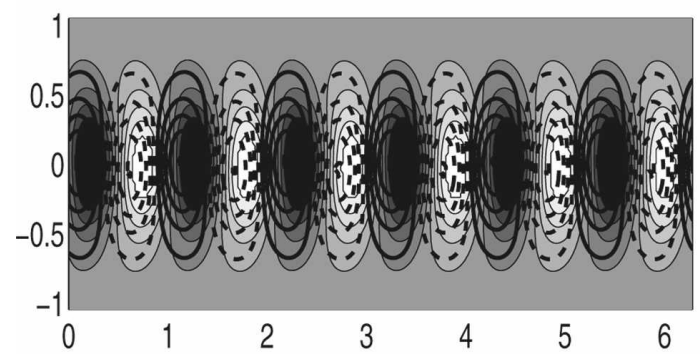

(c) SV with $\mathrm{E}_{-} 0=0.5$ at optimization time

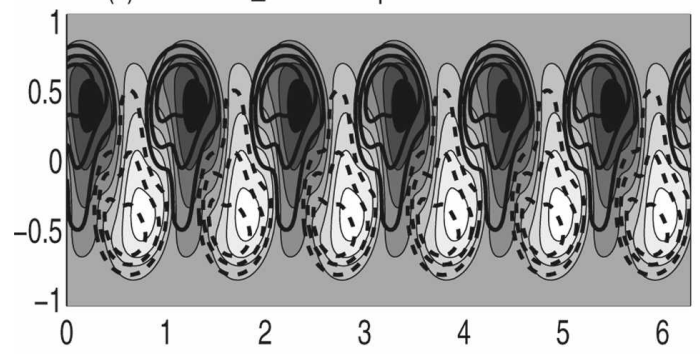

(b) SV with $E_{-} 0=5 \times 10^{\wedge}-2$ at optimization time

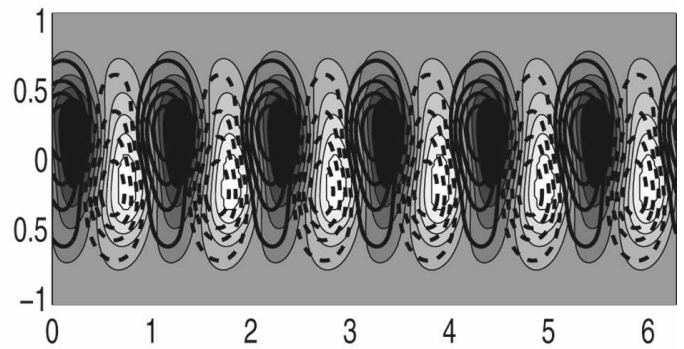

(d) $S V$ with $E_{-} 0=5$ at optimization time

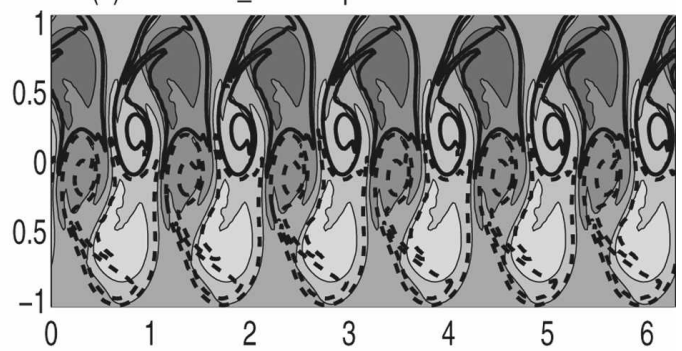

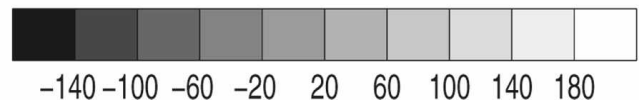

FIG. 2. Snapshots at final time $\left(t=T_{\mathrm{opt}}\right)$ of the potential vorticity of the leading singular vector with initial energy $E_{0}=$ (a) $5 \times 10^{-4}$, (b) $5 \times 10^{-2}$, (c) 0.5 , (d) 5 using the fully nonlinear equations. Potential vorticity has been nondimensionalized by $\sqrt{E_{0}}$. Contours have the same definition as in Fig. 1.

$q_{i}(t)$ divided by an eddy time scale. A typical eddy time scale is the root-mean-square of relative vorticity $\zeta_{\mathrm{rms}}(t)$, giving $J(\psi, q) \approx \zeta_{\mathrm{rms}}(t) q_{i}(t)$. Nonlinear terms will become important when

$$
\lambda \approx \zeta_{\text {rms }}\left(T_{\text {opt }}\right) .
$$

Taking the square of this relation and using $\zeta_{\text {rms }}\left(T_{\text {opt }}\right) \approx$ $\zeta_{\text {rms }}(t=0) \exp \left(\lambda T_{\text {opt }}\right)$, we obtain

$$
\lambda^{2} \approx E_{0} \frac{\zeta_{\mathrm{rms}}^{2}(t=0)}{E_{0}} \exp \left(2 \lambda T_{\mathrm{opt}}\right)
$$

or

$$
E_{0} \approx \frac{E_{0}}{\zeta_{\mathrm{rms}}^{2}(t=0)} \lambda^{2} \exp \left(-2 \lambda T_{\mathrm{opt}}\right)
$$

Because the amplification rate is equal to $\exp \left(2 \lambda T_{\text {opt }}\right)$, and $\zeta_{\mathrm{rms}} / \sqrt{E_{0}} \approx 7.63$ for the leading $\mathrm{SV}$, we find a value for $E_{0}$ of $2.5 \times 10^{-2}$, which gives a relative agreement with Fig. 3.

\section{b. NLSV evolution}

We now turn to the characteristics of the NLSV. First, we can check how the amplification rates of the NLSV and the leading SV compare in the nonlinear model as a function of initial energy. Figure 3 shows that the growth rate of the NLSV is systematically larger than for the leading SV, as it must be. The figure also reveals that the maximum of amplification of the NLSV is reached for very small initial energies, that is, when the evolution of perturbations is linear. When the

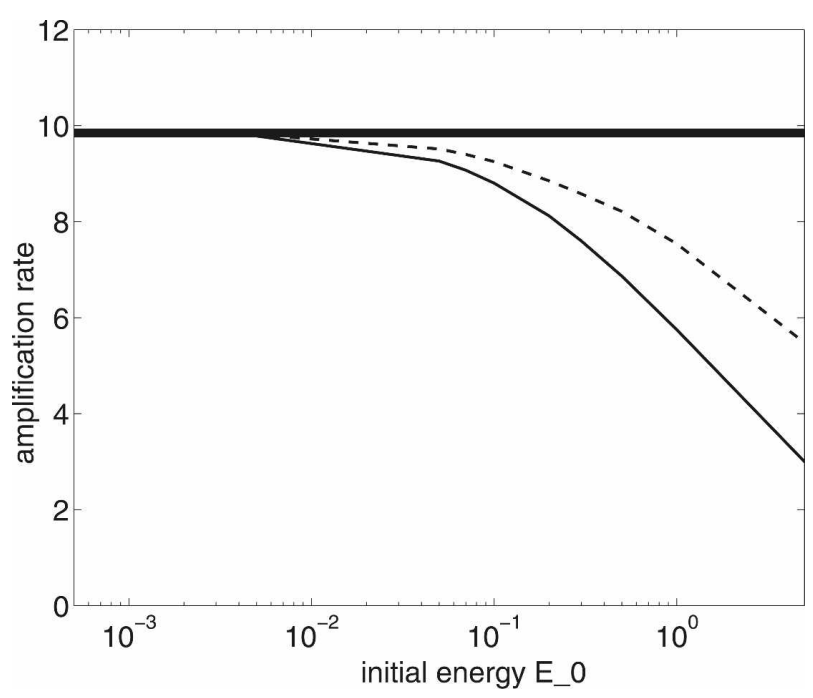

FIG. 3. Amplification rates for SV (solid line) and the NLSV (dashed line) in the nonlinear model as a function of initial energy $E_{0}$. The thick line represents the amplification rate of the SV in the linear model. 
(a) NLSV with $E_{-} 0=5 \times 10^{\wedge}-4$ at initial time

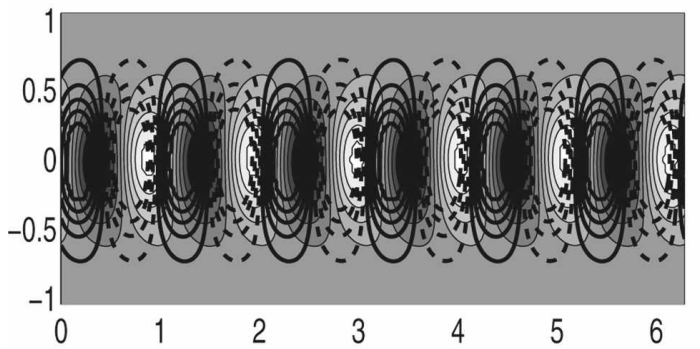

(c) NLSV with $E_{-} 0=0.5$ at initial time

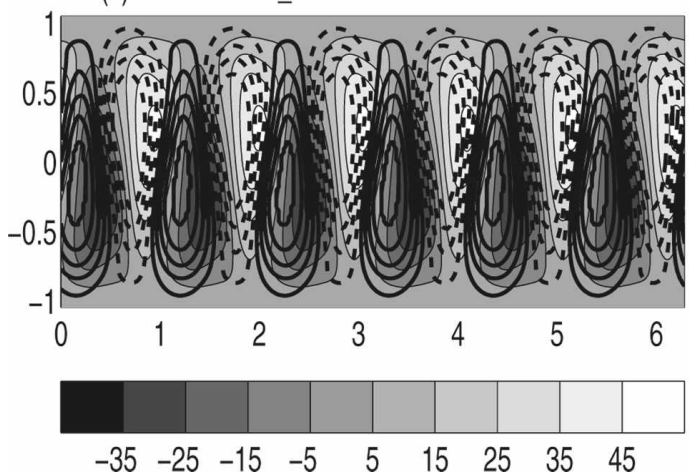

(b) NLSV with $\mathrm{E}_{-} 0=5 \times 10^{\wedge}-2$ at initial time

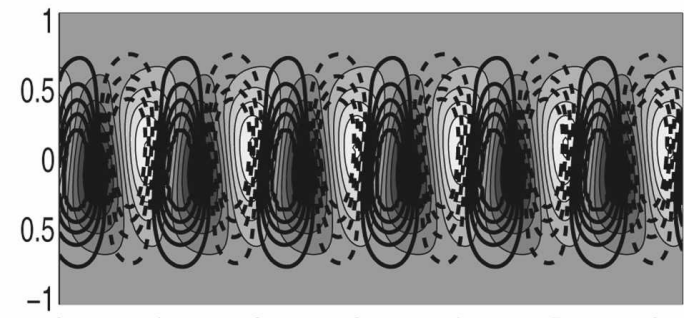

(d) NLSV with $\mathrm{E}_{-} \mathrm{O}=5$ at initial time

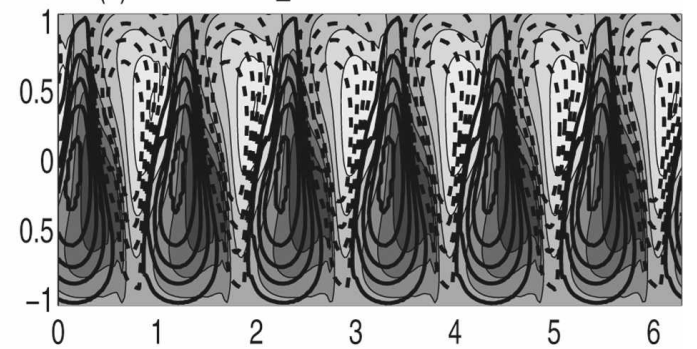

FIG. 4. Potential vorticity of the NLSV (total energy norm) at initial time for different initial energies: $E_{0}=($ a) $5 \times 10^{-4}$, (b) $5 \times 10^{-2}$, (c) 0.5 , (d) 5 . Potential vorticity has been nondimensionalized by $\sqrt{E_{0}}$. Contours have the same definition as in Fig. 1.

initial energy is increased, the NLSV is able to maintain a substantial amplification rate in the nonlinear model even for $E_{0}=0.1$. For this problem [in contrast to situations examined by Duan et al. (2004) and Mu et al. (2004)], nonlinearities systematically inhibit the growth of perturbations.

Figure 4 shows the initial spatial structure of the NLSVs for different initial energies. A comparison of Fig. 4a with Fig. 1b reveals that for small energies, the NLSV and SV have very similar spatial structures, indicating that the method is able to find the global maximum. (We have checked that, when initialized with random initial conditions, the algorithm that computes the NLSV converges toward the leading SV.) When the initial energy is increased, the structures of the NLSV and SV at initial time begin to differ (cf. different panels of Fig. 4). An asymmetry in the initial location of positive and negative PV anomalies can be observed: positive upper-layer and negative lower-layer PV anomalies tend to be on the poleward side of the jet, while opposite anomalies are on the equatorward side of the jet. This asymmetry cannot exist for the SV because there is a symmetry in $y /-y$ in the linear equations of the system (4a), (4b). Also, an increase in the latitudinal extension of the NLSVs can be observed as initial energy is increased. Overall, the spatial field is dominated by large scales and the zonal wavenumber 6 dominates. At the end of the optimization time, for small values of $E_{0}$, we obtain a structure similar to the SV (cf. Figs. 5a and 2a). When the initial energy is increased, we observe that the upper- and lower-layer PV extrema of the NLSV move essentially poleward or equatorward (Figs. 5b-d), similarly to the SV case. For $E_{0}=5$, and contrary to the SV nonlinear evolution, the NLSVs do not form coherent vortices (cf. Figs. 5d and 2d). Another difference is that PV extrema remain vertically aligned for the NLSV at $t=T_{\text {opt }}$ while the PV extrema move in different directions for the leading SV in the nonlinear model. Thus the degree of nonlinearity seems much reduced for the NLSV compared to the SV in the nonlinear model, even for large initial energy. In addition, the NLSV structures are more efficient at carrying heat poleward than SV in the nonlinear model.

\section{Interpretation}

It is necessary to explain the physical mechanisms that differentiate the NLSV from the leading SV, in particular in terms of growth. In the nonlinear evolution, two different mechanisms can be invoked. First, nonlinear dynamics result in wave-mean flow interactions. Perturbations develop through the instability of 
(a) NLSV with $E \_0=5 \times 10^{\wedge}-4$ at optimization time

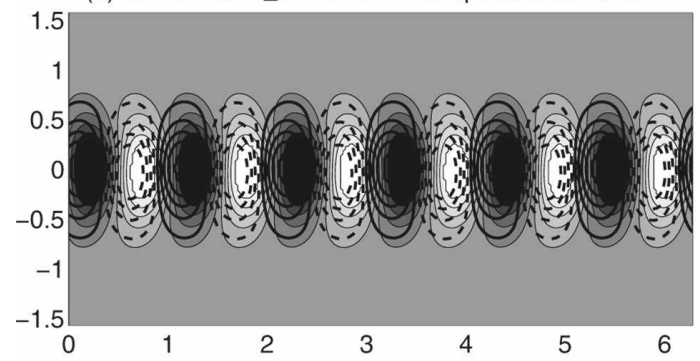

(c) NLSV with E_0 $=0.5$ at optimization time

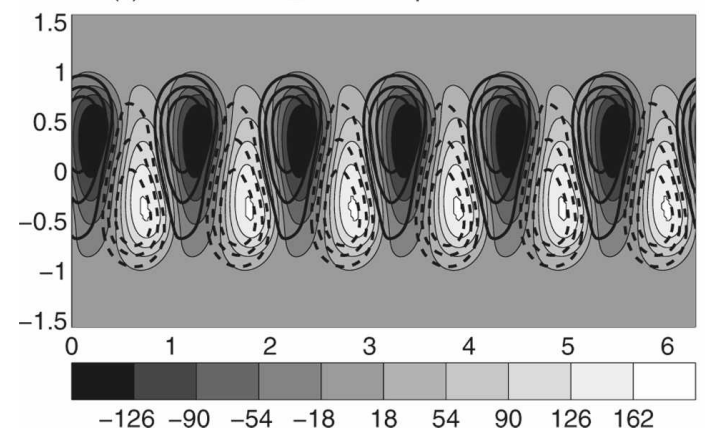

(b) NLSV with $E_{-} 0=5 \times 10^{\wedge}-2$ at optimization time

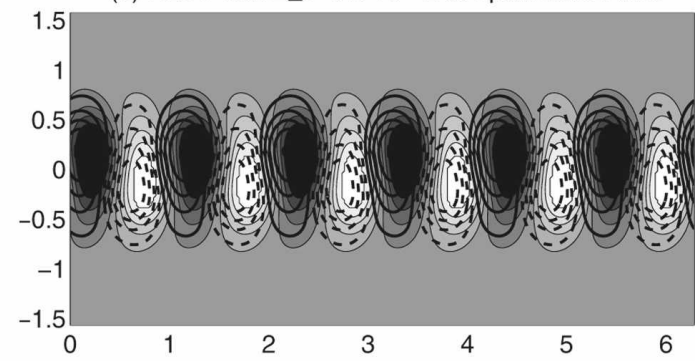

(d) NLSV with $E \_0=5$ at optimization time

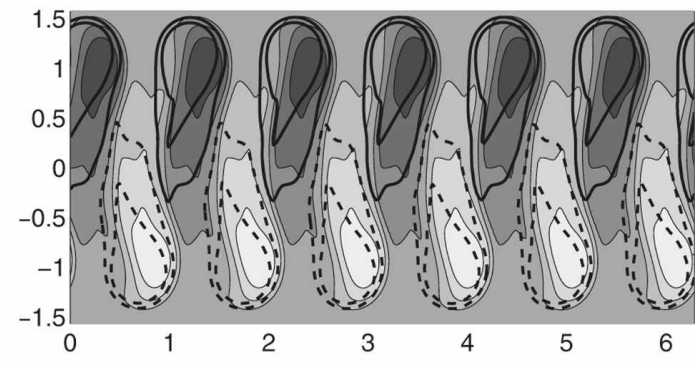

FIG. 5. Potential vorticity of the NLSV (total energy norm) at optimization time $T_{\mathrm{op} t}=0.3$ for different initial energies: $E_{0}=$ (a) $5 \times 10^{-4}$, (b) $5 \times 10^{-2}$, (c) 0.5 , (d) 5 . Potential vorticity has been nondimensionalized by $\sqrt{E_{0}}$. Contours have the same definition as in Fig. 1.

the basic jet and give back their energy to the zonally averaged jet. This can weaken the jet and diminish the shear through the mechanism of baroclinic adjustment. As a result, perturbations will extract less energy from the zonally averaged flow and the perturbation growth will be limited. Second, wave-wave interactions can lead to the development of vortices and to a smaller energy extraction. We will show that the spatial structure of the NLSV adapts to counteract these different interactions, resulting in a larger amplification of the NLSV compared to the SV.

\section{a. Zonal-mean shear of the NLSV}

One important characteristic of nonlinear regimes such as the one studied here is the strong interactions between the perturbations and the large-scale flow. In our setting, it is instructive to decompose the potential vorticity of the NLSV into a zonal mean $\langle q\rangle_{x}$ and a deviation (or eddy part) $q^{\prime}$ such that

$$
q_{i}(x, y, t)=\left\langle q_{i}\right\rangle_{x}(y, t)+q_{i}^{\prime}(x, y, t)
$$

where $\left\langle q_{i}^{\prime}(x, y, t)\right\rangle_{x}=0$, with \langle\rangle$_{x}$ denoting the zonal mean and $i=1,2$. Using the decomposition given by (5) in (3a) and (3b), we can separate the time evolution of the zonal-mean $\left\langle q_{i}\right\rangle_{x}$ and the deviation $q_{i}^{\prime}$ by

$$
\begin{aligned}
\partial_{t} q_{i}^{\prime}= & -\beta \partial_{x} \psi_{i}^{\prime}-J\left(\Psi_{i}+\left\langle\psi_{i}\right\rangle_{x}, q_{i}^{\prime}\right)-J\left(\psi_{i}^{\prime}, Q_{i}+\left\langle q_{i}\right\rangle_{x}\right) \\
& -\left[J\left(\psi_{i}^{\prime}, q_{i}^{\prime}\right)-\left\langle J\left(\psi_{i}^{\prime}, q_{i}^{\prime}\right)\right\rangle_{x}\right] \text { and } \\
\partial_{t}\left\langle q_{i}\right\rangle_{x}= & -\left\langle J\left(\psi_{i}^{\prime}, q_{i}^{\prime}\right)\right\rangle_{x}=-\partial_{y}\left\langle v_{i}^{\prime} q_{i}^{\prime}\right\rangle_{x}
\end{aligned}
$$

The first equation reveals that the energy of the nonzonal perturbations comes from the instability of the basic flow $\left(\Psi_{i}\right.$ and $\left.Q_{i}\right)$ and the zonal-mean flow $\left(\left\langle\psi_{i}\right\rangle_{x}\right.$ and $\left\langle q_{i}\right\rangle_{x}$ ). The last term in the right-hand side of (6a) is due to the self-interactions (or wave-wave interactions). The second equation is the standard wave-mean flow interaction term, which reveals that the eddies retroact on the mean flow (i.e., the zonally averaged flow) through the meridional PV transport, or the divergence of Eliassen-Palm flux (Edmon et al. 1980; Shepherd 1983). This retroaction is important because it modifies the properties of the large-scale flow and in turn this impacts the perturbation growth in (6a).

The time evolution of the SV or the NLSV can be described in different stages (Pedlosky 1964). Initially, perturbations $\left(q^{\prime}\right)$ develop through the instability of the basic flow. During this stage, the linear approximation is valid because the amplitude of the perturbations is small so that the term $J\left(\psi_{i}^{\prime}, q_{i}^{\prime}\right)-\left\langle J\left(\psi_{i}^{\prime}, q_{i}^{\prime}\right)\right\rangle_{x}$ in $(6 a)$ is small (not shown). Then, the eddies modify the mean jet through the poleward advection of heat leading to a 
(a)

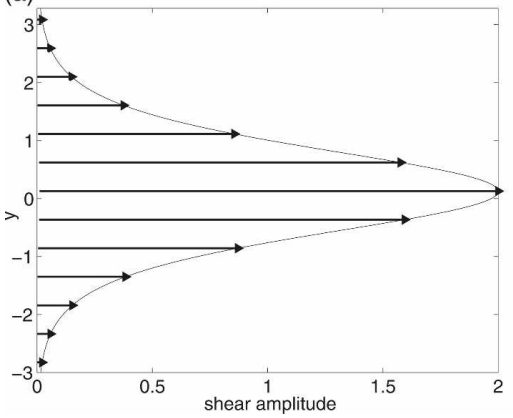

(b)

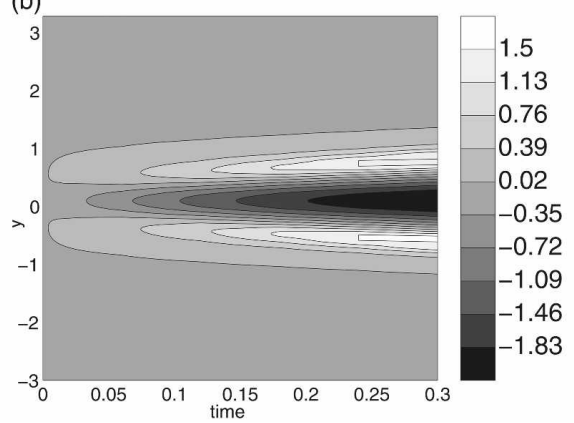

(c)

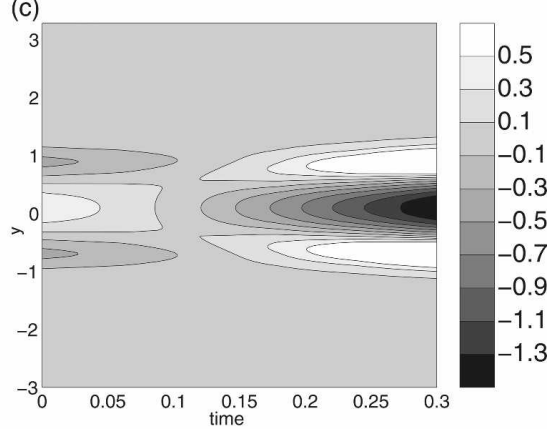

FIG. 6. (a) Zonal-mean shear of the basic jet. Zonal-mean shear $\left\langle u_{1}-u_{2}\right\rangle_{x}$ as a function of time (abscissa) and $y$ (ordinate), for the (b) SV in the nonlinear model and (c) NLSV. For these cases $E_{0}=0.5$.

modification of $\langle q\rangle_{x}$ through (6b). This is apparent in Fig. 6b, which shows the evolution in time of the zonal shear $\left\langle u_{1}-u_{2}\right\rangle_{x}$ for the leading SV. A strong zonal shear opposite to the basic shear develops through time. In response, the growth rate of instability (given by the total zonal-mean shear) should diminish (Gutowski 1985; Nakamura 1999). Another effect is that the nonlinear term $J\left(\psi^{\prime}, q^{\prime}\right)-\left\langle J\left(\psi^{\prime}, q^{\prime}\right)\right\rangle_{x}$ modifies the waves so that they may break and form vortices. This last stage is apparent for the SV time evolution, while it seems absent for the NLSV (cf. Figs. 2d and 5d).

An important difference between the leading SV and the NLSV at initial time is that the NLSV possesses a zonal-mean shear in the same direction as the basic jet, but with retrograde jets on both sides (Fig. 6c). This reinforces the basic jet so that the final mean shear of the NLSV is smaller than in the SV case. The asymmetric meridional structure of the PV of the NLSV (as shown in Fig. 4) is indeed related to the presence of this zonal component. The thermal wind balance implies that the zonal shear is associated with a meridional temperature gradient that reinforces positive temperature anomalies equatorward and negative anomalies poleward at initial time. Another confirmation of the importance of this mean shear is provided by the comparison with experiments for which the initial zonalmean shear is reversed or suppressed. In these two cases, the amplification of the structure is smaller than for the SV (Table 2). In contrast, changing SV into its opposite has no influence on the amplification rate (not shown). This demonstrates the important role played by the total mean shear (basic state plus perturbation) in the nonlinear development of the instability.

To confirm the importance of the initial zonal-mean shear, it is instructive to decompose the energetics into a zonal and an eddy part. Multiplying (6a) by $-\psi_{i}^{\prime}$, (6b) by $-\left\langle\psi_{i}\right\rangle_{x}$, horizontally averaging each equation, and summing over the layers, we obtain

$$
\begin{aligned}
\partial_{t} \mathrm{ETE}= & \sum_{i=1}^{2}\left\langle u_{i}^{\prime} v_{i}^{\prime} \partial_{y}^{2} \Psi_{i}\right\rangle+\sum_{i=1}^{2}\left\langle u_{i}^{\prime} v_{i}^{\prime} \partial_{y}^{2}\left\langle\psi_{i}\right\rangle_{x}\right\rangle \\
& -\lambda^{-2}\left\langle v_{1}^{\prime}\left(\psi_{1}^{\prime}-\psi_{2}^{\prime}\right) \partial_{y}\left(\Psi_{1}-\Psi_{2}\right)\right\rangle \\
& -\lambda^{-2}\left\langle v_{1}^{\prime}\left(\psi_{1}^{\prime}-\psi_{2}^{\prime}\right) \partial_{y}\left\langle\psi_{1}-\psi_{2}\right\rangle_{x}\right\rangle \quad \text { and } \\
\partial_{t} \mathrm{ZTE}= & -\left\langle u_{1}^{\prime} v_{i}^{\prime} \partial_{y}^{2}\left\langle\psi_{1}\right\rangle_{x}\right\rangle-\left\langle u_{2}^{\prime} v_{2}^{\prime} \partial_{y}^{2}\left\langle\psi_{2}\right\rangle_{x}\right\rangle \\
& +\lambda^{-2}\left\langle v_{1}^{\prime}\left(\psi_{1}^{\prime}-\psi_{2}^{\prime}\right) \partial_{y}\left\langle\psi_{1}-\psi_{2}\right\rangle_{x}\right\rangle,
\end{aligned}
$$

where

$$
\begin{aligned}
\mathrm{ETE}= & \frac{1}{2}\left\langle\left(\partial_{x} \psi_{1}^{\prime}\right)^{2}+\left(\partial_{y} \psi_{1}^{\prime}\right)^{2}+\left(\partial_{x} \psi_{2}^{\prime}\right)^{2}+\left(\partial_{y} \psi_{2}^{\prime}\right)^{2}\right\rangle \\
& +\frac{1}{2} \lambda^{-2}\left\langle\left(\psi_{1}^{\prime}-\psi_{2}^{\prime}\right)^{2}\right\rangle \text { and } \\
\mathrm{ZTE}= & \frac{1}{2}\left\langle\left(\partial_{y}\left\langle\psi_{1}\right\rangle_{x}\right)^{2}+\left(\partial_{y}\left\langle\psi_{2}\right\rangle_{x}\right)^{2}\right\rangle \\
& +\frac{1}{2} \lambda^{-2}\left\langle\left(\left\langle\psi_{1}-\psi_{2}\right\rangle_{x}\right)^{2}\right\rangle .
\end{aligned}
$$

ETE is the eddy part of the total energy (kinetic plus available potential energies) and ZTE is the zonal part. The decomposition of the total energy [Eqs. (7a), (7b)] reveals that the eddy energy can grow through barotropic and baroclinic extraction from the basic flow $\Psi$ [terms 1 and 3 on the right-hand side of (7a)]. Then this energy can be transferred to the zonal part through terms 2 and 4 of (7a). Figure 7a shows the ETE production [right-hand side of (7a)] for both the SV and NLSV in the nonlinear model for $E_{0}=0.5$. The growth

TABLE 2. Amplification rates for different initial conditions based on the NLSV; $\langle q\rangle_{x}$ is the zonal-mean part of the NLSV and $q^{\prime}$ the deviation. For each type of structure, there was no rescaling of initial energy.

\begin{tabular}{cccc}
\hline \hline Structure & $\langle q\rangle_{x}+q^{\prime}$ & $-\langle q\rangle_{x}+q^{\prime}$ & $+q^{\prime}$ \\
\hline Amplification & 8.21 & 6.17 & 7.73
\end{tabular}


(a)

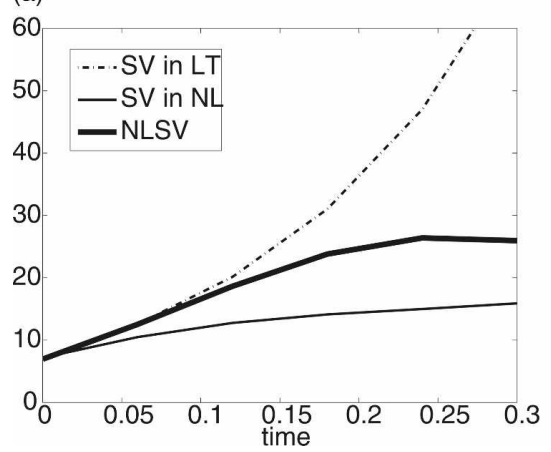

(b)

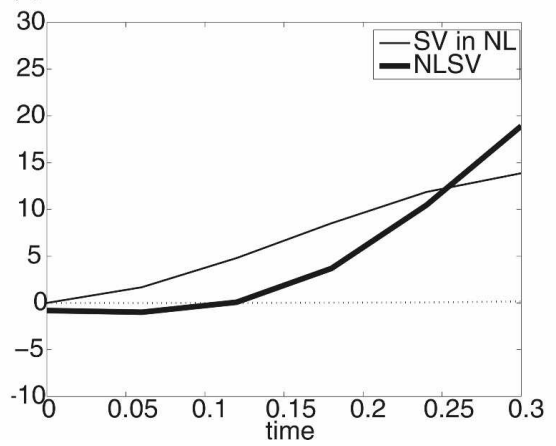

(c)

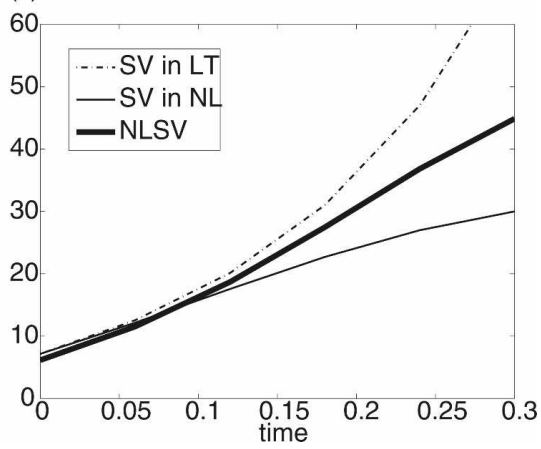

FIG. 7. (a) Production of eddy total energy over time following Eq. (7a). The dash-dotted (thin solid) line represents the energy production for the SV in the linear (nonlinear) model. The thick solid line represents the production for the NLSV. (b) Production of zonal total energy over time following Eq. (7b). The thin solid (thick solid) line represents the production for the SV (NLSV). (c) Extraction of EAPE from the basic jet [term 3 in Eq. (7a)]. Curves have same definition as in (a). The initial energy for each case is $E_{0}=0.5$.

rate of ETE of the SV saturates very rapidly in time compared to the linear case. This can be attributed to the steady growth of the zonal part (Fig. 7b). On the contrary, the zonal part of the NLSV remains small until $t=0.1$ (Fig. 7b) and the eddy part has a growth rate as strong as the linear SV until that time (Fig. 7a). For the particular basic jet we use, we have found that the barotropic terms remain small over the entire time evolution and the energy production essentially comes from the eddy available potential energy [EAPE; $\left.\lambda^{-2}\left\langle\left(\psi_{1}^{\prime}-\psi_{2}^{\prime}\right)^{2}\right\rangle / 2\right]$ production. The impact of the eddyeddy interaction can be assessed by examining the energy extraction terms 3 and 4 of (7a). Figure 7c shows a smaller extraction of EAPE from the basic flow for the SV than for the NLSV after $t=0.1$. This means that after that time, wave-wave interactions have made the SV less efficient in extracting energy. On the contrary, the NLSV has an extraction that compares well with the linear SV case.

\section{b. Meridional extension of the NLSV}

One could think that the presence of the initial zonal shear in the NLSV can explain most of the behavior of the NLSV. However, in addition to this zonal shear, the NLSV is more elongated in the meridional direction than the SV and this phenomenon needs to be examined. To see if this effect is important for the amplification, we have conducted experiments where we initialized a structure with the same profile in $x$ as the SV and with an exponential decay in the $y$ direction, such that

$$
\begin{aligned}
q(x, y, t=0)= & \left\langle q_{\mathrm{NLSV}}\right\rangle_{x}(y, t=0) \\
& +\gamma q_{\mathrm{SV}}(x, y=0, t=0) \exp \left(-a y^{2}\right),
\end{aligned}
$$

where $a^{-1 / 2}$ sets the meridional decay set and $\gamma$ is an adjustable parameter so that the initial energy is $E_{0}=$ 0.5. Different profiles are represented in Fig. 8. We have verified that the exponential function in $y$ fits the SV well for $a=6$ (not shown). The differences in behavior for different values of $a$ allow us to interpret the effect of the meridional extension of the structure. Table 3 shows that adding the zonal shear to the SV increases the amplification (7.35 against 6.86 ) but this is still smaller than the NLSV amplification (8.21). We conclude from this that the zonal shear of the SV alone is not sufficient to increase the growth rate of the SV compared to the NLSV growth rate for the same total

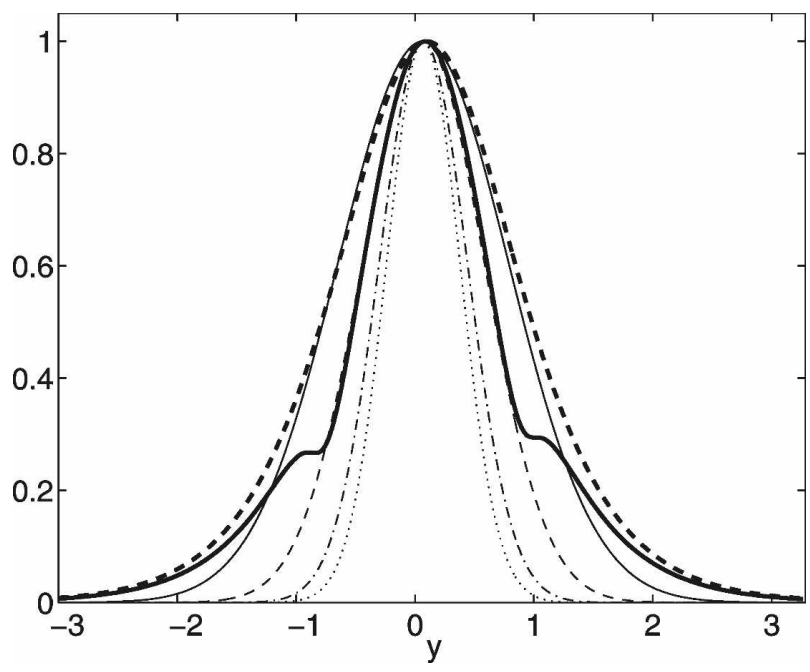

FIG. 8. Profile of $\exp \left(-a y^{2}\right)$ as a function of $a=1$ (thin solid line), $a=2$ (thin dashed line), $a=4$ (dash-dotted line), $a=6$ (dotted line). The thick and dashed curve is the basic shear $-\partial_{y} \Psi$ and the thick and solid line is the total zonal-mean shear $-\partial_{y}(\Psi+$ $\left.\langle\psi\rangle_{x}\right)$ for the NLSV at $E_{0}=0.5$. All quantities were renormalized. 
TABLE 3. Amplification rate for the experiment with a different meridional extension for the SV with the zonal mean of the NLSV [see (8)].

\begin{tabular}{ccccc}
\hline \hline$a$ & 6 & 4 & 2 & 1 \\
\hline Amplification & 7.35 & 7.76 & 8.09 & 7.80 \\
\hline
\end{tabular}

energy. It is also necessary to modify the nonzonal structure to obtain the largest growth rate. Indeed, it is possible to obtain an amplification (8.09) that is close to the NLSV (8.21) for a meridional extension parameter $a=2$. From Table 3 , we see that there is an optimal extension for the amplification after which the amplification rate decays. The reason is that when the structures are too broad, they cannot extract energy from the basic jet because the basic jet is meridionally confined.

The existence of an optimal extension suggests that there is a link between the optimal structure and the shape of the jet. Indeed, Pedlosky and Klein (1991) have shown that for weakly nonlinear baroclinic unstable flows, the meridional variation of the basic shear can strongly modify the amplification of perturbations. For perturbations having the same meridional structure as the basic shear, wave-mean flow interactions are unable to arrest the growth of the waves. It is interesting to compare the exponential profile of the different solutions of (8) with the basic shear $-\partial_{y} \Psi$ and the total shear of the NLSV $-\partial_{y}\left(\Psi+\langle\psi\rangle_{x}\right)$. Figure 8 shows these quantities and demonstrates a good agreement between the total shear and the exponential profile for $a=2$. Surprisingly, this corresponds to the case of maximum amplification. Therefore, we are able to confirm the mechanism of Pedlosky and Klein (1991) in our fully nonlinear setting. It reveals that the meridional extension of the NLSV is tightly linked to the initial shear present in the NLSV so that wave-mean flow interactions cannot arrest the growth of the perturbations.

The presence of the zonal-mean shear and the meridional elongation of the NLSV has an impact on the nonlinearities of the system because it reduces the initial amplitude of $q_{i}^{\prime}$. For a given total energy $E_{0}=0.5$, the eddy energy is only 0.45 , that is, smaller than the eddy energy of the SV (equal to $E_{0}$ ). Also, the meridional broadening of the NLSV favors a smaller perturbation maximum for the same energy. This reduces the importance of nonlinearities in the term $J\left(\psi^{\prime}, q^{\prime}\right)$ $\left\langle J\left(\psi^{\prime}, q^{\prime}\right)\right\rangle_{x}$ in the case of the NLSV in contrast to the SV. To verify this, we can examine a model where the nonlinear term $J\left(\psi_{i}^{\prime}, q_{i}^{\prime}\right)-\left\langle J\left(\psi_{i}^{\prime}, q_{i}^{\prime}\right)\right\rangle_{x}=0$ in (6a). The evolution equations for this weakly nonlinear model are

$$
\begin{aligned}
\partial_{t}\left\langle q_{i}\right\rangle_{x} & =-\left\langle J\left(\psi_{i}^{\prime}, q_{i}^{\prime}\right)\right\rangle_{x} \text { and } \\
\partial_{t} q_{i}^{\prime} & =-J\left(\Psi_{i}+\left\langle\psi_{i}\right\rangle_{x}, q_{i}^{\prime}\right)-J\left(\psi_{i}^{\prime}, Q_{i}+\left\langle q_{i}\right\rangle_{x}\right) .
\end{aligned}
$$

As shown in Table 1, the amplifications of the NLSV and the leading SV in the weakly nonlinear model are quite close to the amplifications in the nonlinear model for $E_{0}=0.5$. We found that this is not true for larger initial energy $E_{0}$ (not shown). This can be expected because nonlinearities may be stronger in that case.

\section{Generalization}

Singular vectors are known to be sensitive to the choice of the norm and to the optimization time. We thus expect that NLSVs will also exhibit a dependence on these parameters. However, the arguments developed above that explain the difference between SV and NLSV were expressed in terms of energetics, and we may be inclined to think that they may still be valid when changing these parameters.

\section{a. Other norms}

Joly (1995) and Palmer et al. (1998), among others, have examined the effect of the norm on singular vectors. They have found that SVs computed using total energy or streamfunction variance as norm are spatially confined whereas SVs computed using potential enstrophy have a larger spatial extent. The reason is that the potential enstrophy gives more weight to small scales as a norm. It will thus favor large scales at initial time and smaller scales will develop during the time evolution, leading to an amplification of the norm. The situation is the opposite for the streamfunction variance norm that gives more weight to large scales as a norm and selects smaller scales at initial time. Moreover, the potential enstrophy norm is more barotropic because it is less sensitive to the Orr mechanism (Joly 1995; Rivière et al. 2001; Kim and Morgan 2002; Heifetz and Methven 2005). We therefore tried to compute the leading SV and different NLSVs for different norms. We can first examine the total potential enstrophy norm

$$
Z(q)=\frac{1}{2}\left\langle q_{1}^{2}+q_{2}^{2}\right\rangle
$$

We use the same optimization time $T_{\text {opt }}=0.3$ as for the total energy norm. Figure 9 shows that the singular vector that has evolved in the nonlinear model has an amplification rate $Z\left[q\left(t=T_{\mathrm{opt}}\right)\right] / Z[q(t=0)]$ that rapidly decreases between $Z_{0}=Z[q(t=0)]=1$ and $Z_{0}=$ 100. For these potential enstrophies, the NLSV has a significantly larger amplification rate than the SV. The spatial structure of the SV (or the NLSV with very 


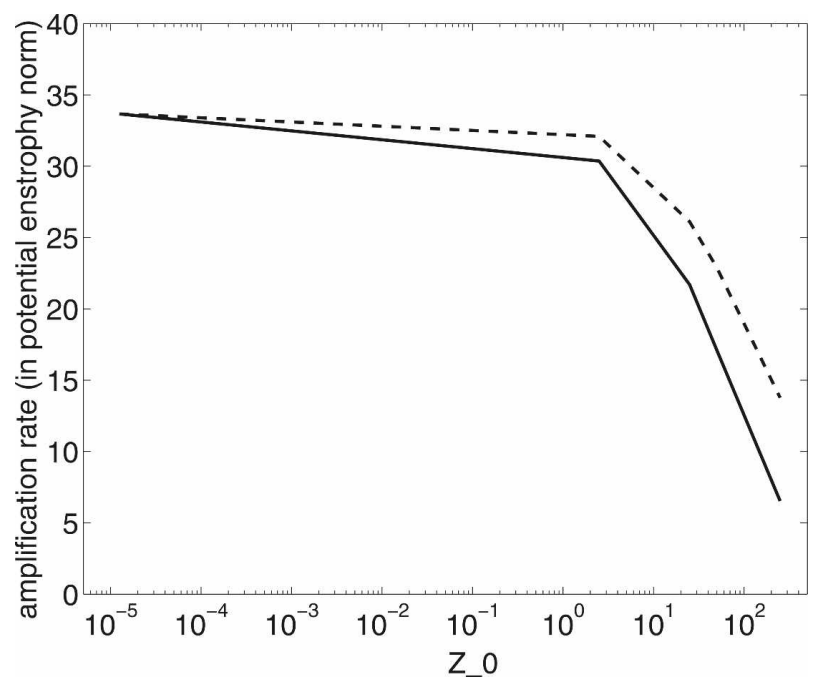

FIG. 9. Amplification rate for SV (solid line) and the NLSV (dashed line) for the potential enstrophy norm in the nonlinear model as a function of initial potential enstrophy $Z_{0}$.

small $Z_{0}$ ) has a zonal wavenumber 4 that emerges (Fig. 10a). This is a known result because SVs peak at larger scales for the potential enstrophy norm than for the total energy norm (Rivière et al. 2001). One noticeable feature, different from the total energy norm, is the almost barotropic character of the PV perturbations. When increasing the initial potential enstrophy $Z_{0}$, we observe the meridional shift of positive and negative PV maxima (Figs. 10b,c). This was also apparent for the total energy norm. This displacement of the structures is due to the presence of a mean shear that increases with $Z_{0}$ (not shown). Also, the NLSV structure spreads meridionally (Fig. 10d) and is reminiscent of results for the total energy norm (cf. with Fig. 4c). At the optimization time, we see that the NLSV structures tend to move meridionally (Figs. 11b,c,d) whereas the SV moves zonally in the linear model (Fig. 11a). The PV maxima are vertically aligned with PV minima, which means that temperature dominates relative vorticity in the PV. For very large potential enstrophy, we see that vortices begin to emerge at the end of the optimization time (Fig. 11d). This norm seems less effective in inhibiting vortex formation. However, the general characteristics of potential enstrophy NLSV are similar to those of the total energy NLSV. This indicates that the mechanisms of nonlinear amplification are very similar to the mechanisms for the total energy norm.

Another norm that can be considered is the streamfunction variance

$$
P(q)=\frac{1}{2}\left\langle\psi_{1}^{2}+\psi_{2}^{2}\right\rangle
$$

Linear singular vectors are well defined with this norm. We have made several tests to compute NLSVs with this norm but we were not able to make the algorithm converge for $P(q)$ large enough so that nonlinearities limit amplification. During the process of optimization, structures at the smallest possible scales begin to emerge and become dominant in the spectrum (not shown). We attribute this problem to the choice of the norm: as stated before, the streamfunction variance puts more weight on the development of larger scales between $t=0$ and $t=T_{\mathrm{opt}}$. Therefore, the optimization algorithm tries to find an initial structure that possesses energetic small scales that it will make grow in size as time evolves. In the linear setting, there is no scale interaction and a particular mode is selected. When nonlinear interactions are allowed, the algorithm makes large and small scales interact. As a result, small scales tend to dominate the energy spectrum and the NLSV technique exacerbates these scales. The computation of physically relevant NLSVs in this case is therefore not possible.

\section{b. Optimization time}

As investigated by Rivière et al. (2001) and others, the optimization time has some effect on the singular vector structure. We examine here the dependence of NLSV on $T_{\text {opt }}$ using the total energy norm.

First, we examine the long time limit, taking $T_{\mathrm{opt}}=$ 1.2. In this case, the SV structure has a dominant zonal wavenumber 7 similar to the normal mode. Indeed, at the final time, the leading SV strongly resembles the normal mode (not shown). Its amplification rate is 6297.5 in the linear model and 3555.8 in the nonlinear model for $E_{0}=10^{-3}$. The corresponding NLSV has an amplification rate of 3748.8. The structure of the NLSV in physical space is quite similar to results with $T_{\mathrm{opt}}=$ 0.3. Comparing Figs. 12a and $4 \mathrm{c}$, we see that the initial PV structures are in each case asymmetric with respect to the jet axis, with PV extrema on either side of the jet. A difference is that NLSVs with $T_{\text {opt }}=0.3$ are stretched in a triangular shape, whereas NLSVs with $T_{\mathrm{opt}}=1.2$ have a more rectangular shape. This means that the shear is less intense and weakly shifts the PV extrema. At the end of the optimization time, the PV structures have a stronger horizontal tilt than for $T_{\mathrm{opt}}=$ 0.3 (Fig. 12b).

We now examine the short time limit, taking $T_{\mathrm{opt}}=$ 0.03 . The leading SV has an amplification rate of 1.28 in the linear model. It has a zonal wavenumber 4 (Fig. 13a). In the nonlinear model, the amplification rate is only 1.21 for $E_{0}=100$. We still see the meridional displacement of PV maxima, associated with the development of a negative shear (Fig. 13c). Also, PV tends to 
(a) NLSV for $Z \_0=2.5 \times 10^{\wedge}-5$ at $t=0$

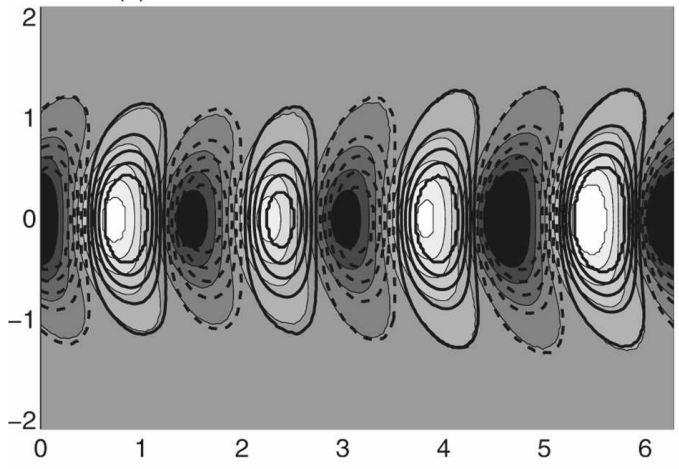

(c) NLSV for $Z \_0=50$ at $t=0$

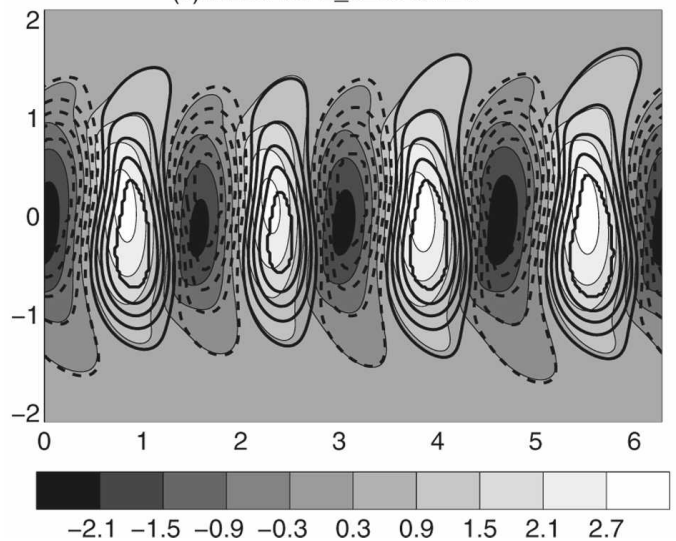

(b) NLSV for Z_ $0=5$ at $t=0$

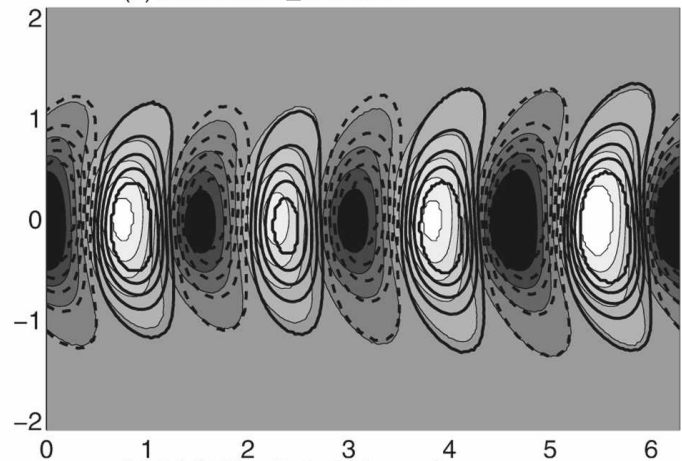

(d) NLSV for $Z$ Z $0=500$ at $t=0$

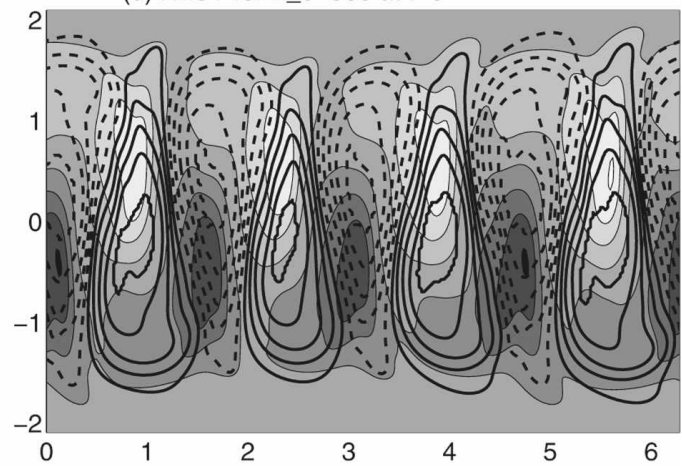

FIG. 10. Potential vorticity of the NLSV (potential enstrophy norm) at initial time for different initial potential enstrophies: $Z_{0}=$ (a) $2.5 \times 10^{-5}$, (b) 5 , (c) 50 , (d) 500 . Potential vorticity has been nondimensionalized by $\sqrt{Z_{0}}$. Contours have the same definition as in Fig. 1.

roll up into vortices even for this small optimization time. The corresponding NLSV has an amplification rate of 1.26 and the structure in physical space (Fig. $13 b)$ has a strong asymmetry in the PV extrema, similar to the case of $T_{\text {opt }}=0.3$. There is still the presence of a positive shear that helps in maintaining the growth rate. The NLSV structure extends meridionally as $E_{0}$ increases. At the final time, the PV structures move meridionally and do not form vortices (Fig. 13d), similar to the case of $E_{\mathrm{op} t}=0.3$.

The amplitude of the shear compared to the nonzonal velocity anomalies seems to depend on the optimization time; it is less intense for longer optimization times. This means that to maintain a growth rate over a long time period, the NLSV structure needs to inhibit the formation of vortices, leading to a broader meridional extension. On the contrary, this effect is less intense for short optimization times and the optimization needs to put more weight on the zonal-mean shear.

\section{Discussion}

This study has revealed how nonlinearities affect baroclinic growth through the use of a new technique called nonlinear singular vector. NLSVs are an extension to the nonlinear regime of singular vectors and were first proposed by $\mathrm{Mu}$ (2000). More specifically, they are perturbations with a given initial energy that maximize the amplification rate over a fixed time in the fully nonlinear system. They can be computed using constrained optimization algorithms.

In the Phillips model of baroclinic instability, it is well known that SVs have a limited growth in the nonlinear model. We showed here that NLSVs are rather similar to SV in their spatial patterns but that they maintain a larger amplification in the nonlinear model. NLSVs differ from the leading SV essentially by the presence of a zonal-mean flow (that is precluded for the SV by the symmetry in the linear equations) and by their broader meridional extension. The zonal-mean flow initially present in the NLSV maintains a strong production of potential energy during the time evolution. This tendency opposes the natural tendency of nonlinearities in baroclinic unstable flows that are responsible for a poleward heat flux that decelerates the mean jet (through the Eliassen-Palm flux). As a result, one can view NLSVs in this baroclinic problem as weakly non- 
(a) NLSV for $Z \_0=2.5 \times 10^{\wedge}-5$ at $t=T \_o p t$

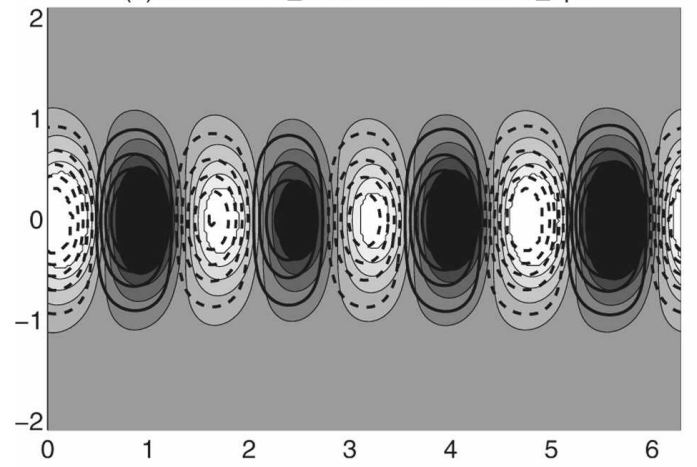

(c) NLSV for Z_0 $=50$ at $t=T \_o p t$

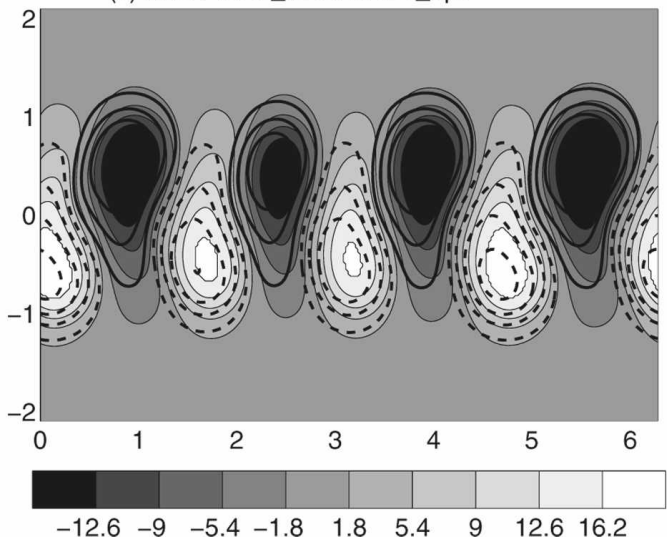

(b) NLSV for $Z \_0=5$ at $t=T \_o p t$

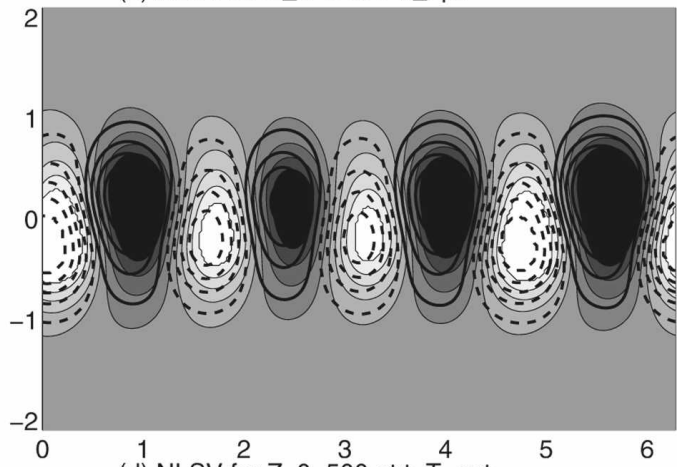

(d) NLSV for $Z$ _ $0=500$ at $t=T_{-}^{4}$ opt

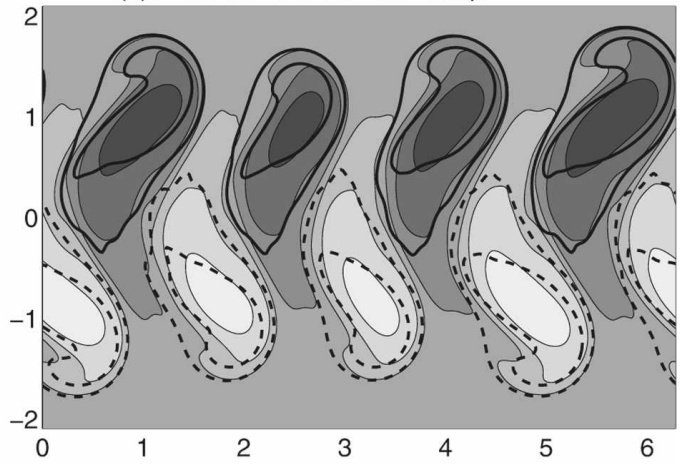

FIG. 11. Potential vorticity of the NLSV (potential enstrophy norm) at optimization time for different initial potential enstrophies: $Z_{0}=$ (a) $2.5 \times 10^{-5}$, (b) 5 , (c) 50 , (d) 500 . Potential vorticity has been nondimensionalized by $\sqrt{Z_{0}}$. Contours have the same definition as in Fig. 1 .

linear structures for which nonlinearities apply to the zonal-mean flow at the first order, similarly to the development of Pedlosky (1964). Another aspect is that NLSVs have a broader meridional extension than SVs. This limits wave-wave interactions and the development of vortices so that the NLSV extracts more energy than the SV during the time evolution. In these simulations, the zonal wavenumber remains the same for the
NLSV as one increases its initial energy. We observed that in other settings (in particular without the $\beta$ effect and with a bottom drag), the zonal wavenumber decreases as $E_{0}$ increases (not shown).

The picture that emerges is that the NLSV modifies the structure of the wave (the nonzonal part of the NLSV) and adds a zonal flow to take the nonlinear baroclinic adjustment into account. The most unstable (a) NLSV at initial time for T_opt $=1.2$

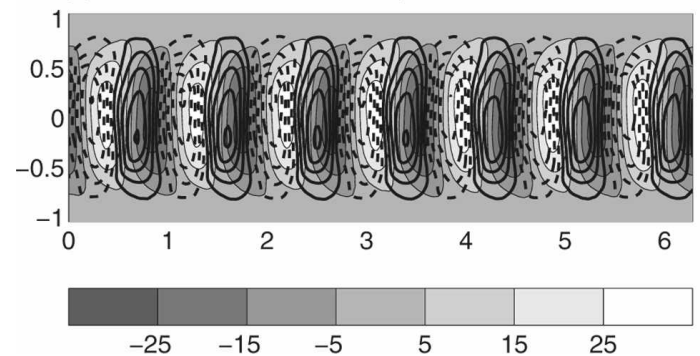

(b) NLSV at final time for T_opt=1.2

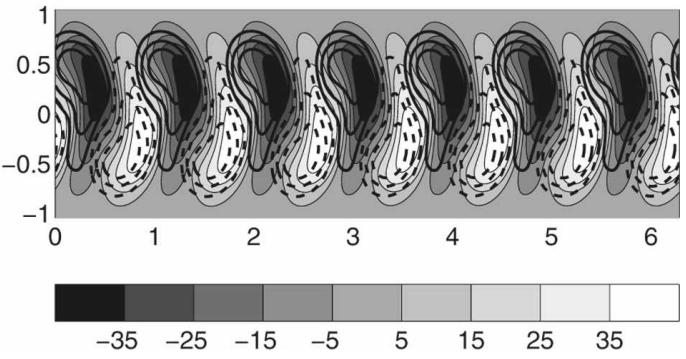

FIG. 12. Potential vorticity of the NLSV (total energy norm) at (a) initial and (b) optimization times for $E_{0}=$ $10^{-3}$ and $T_{\text {opt }}=1.2$. Potential vorticity has been nondimensionalized by $\sqrt{E_{0}}$. Contours have the same definition as in Fig. 1. 
(a) SV at $t=0$ for $T \_o p t=0.03$

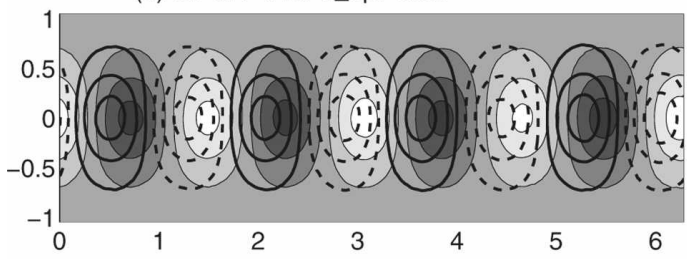

(c) SV at t=T_opt for $\mathrm{T} \_$opt $=0.03$

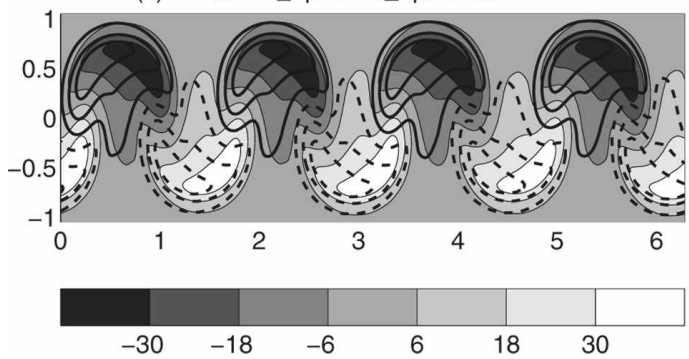

(b) NLSV at $t=0$ for $T \_o p t=0.03$

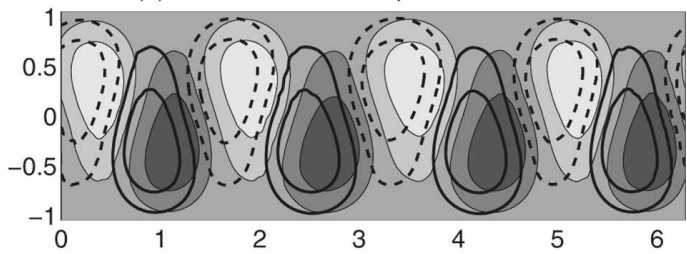

(d) NLSV at t=T_opt for T_opt $=0.03$

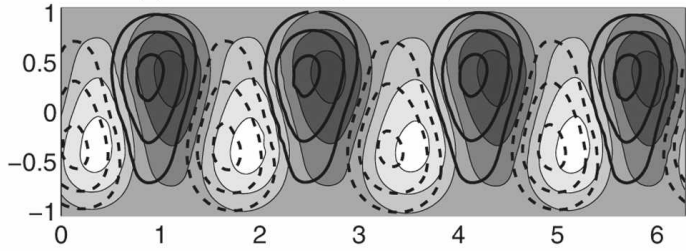

FIG. 13. Potential vorticity of the (a) SV and (b) NLSV at initial time and (c) SV and (d) NLSV at optimization time (total energy norm) for $E_{0}=100$ and $T_{\text {opt }}=0.03$. Potential vorticity has been nondimensionalized by $\sqrt{E_{0}}$. Contours have the same definition as in Fig. 1.

wave will saturate rapidly, so that it is not the wave of largest growth rate over a finite time. Therefore, the NLSV selects a modified wave (which has a different meridional spatial scale but the same zonal scale in our case) that may be less unstable initially, but that will have a larger heat flux over the evolution in time. This behavior is similar to the findings of Pedlosky (1979), Hart (1981), and Cehelsky and Tung (1991), who found a selection of a particular wave, different from the most unstable one, for weakly nonlinear and baroclinic unstable flows.

These optimal nonlinear perturbations are suggestive of bred vectors. Bred vectors are perturbations that have grown in the nonlinear model and are rescaled to a finite energy at a given frequency in time (Toth and Kalnay 1993). Letting the energy amplitude of renormalization tend to zero, one obtains the Lyapunov vector (here the normal mode of the system because our basic flow is steady). We have computed bred vectors for our problem with a renormalization in initial energy, taking $E_{0}=10^{-2}$ and with a time of renormalization equal to $T_{\mathrm{opt}}=0.3$. We have initialized the procedure with the normal mode and we have run the nonlinear model for $100 T_{\mathrm{opt}}$, renormalizing the solution after each $T_{\text {opt }}$ time interval. The solution converges toward a stable structure. The growth rate of the bred vector is only 7.73, that is, much smaller than the growth rate of the SV and the NLSV in the nonlinear model for the same initial energy (see Fig. 3). Figure 14 shows that these structures have PV maxima centered on each side of the basic jet, with patterns resembling those of the leading SV and NLSV at the final time (cf.
Figs. 2 and 5). Indeed, the bred vector has an initial negative shear because the structure has evolved in time. This shear limits the bred vector growth. On the other hand, the NLSV possesses an initial positive shear to counteract this effect and this explains why the NLSV growth is larger.

We believe that the NLSV approach is well suited for other problems, provided that there is a typical length scale that defines the instability or its saturation. If small scales are strongly unstable (such as for the case of convection), the NLSV algorithm may not converge. This is the case in our model when using the streamfunction variance norm for which small scales at initial time are promoted by the NLSV algorithm. We are currently extending the NLSV approach to more realistic situations using a primitive equation model. This will allow us to better understand the role of nonlin-

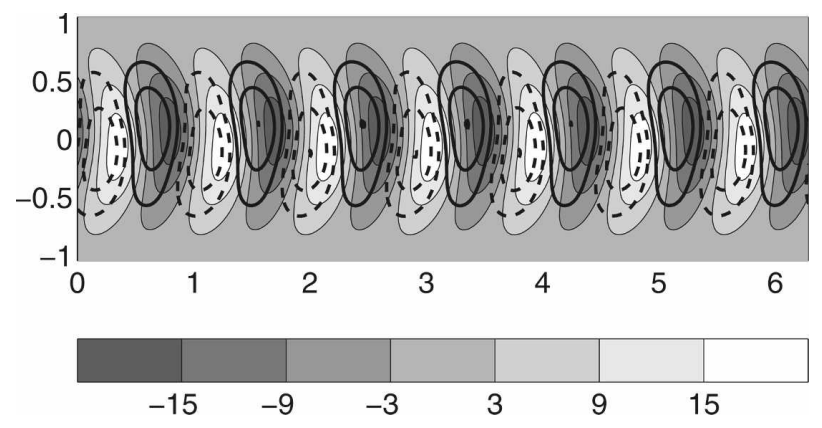

FIG. 14. Potential vorticity of the bred vector for $E_{0}=10^{-2}$ after renormalization. Potential vorticity has been nondimensionalized by $\sqrt{E_{0}}$. Contours have the same definition as in Fig. 1. 
earities in stratified baroclinic unstable flows. Our ultimate goal is to use such a method to study problems for which nonlinearities can be important. Such a situation can arise when taking into account the role of water vapor and latent heat release, which can have a critical impact on moist synoptic systems (Lapeyre and Held 2004). In this case, the precipitation introduces a threshold function and this may not be well handled by the adjoint model (used for the computation of the NLSV). In the framework of four-dimensional variational data assimilation (4DVAR) studies, it was shown that the BFGS method was still working well even in the presence of thresholds (Zou et al. 1993). Two reasons can be invoked: first, the gradient, even if not perfectly defined, remains a good approximation of the search direction; and second, the line-search procedure (used once the search direction has been retrieved and requiring sufficient decrease in the cost function) can allow the algorithm to cross thresholds provided the step size is not too small. Preliminary experiments have been done and have shown some success in finding NLSVs. These results will be reported in a future manuscript.

Acknowledgments. OT acknowledges stimulating discussions with $\mathrm{Mu} \mathrm{Mu}$.

\section{REFERENCES}

Badger, J., and B. J. Hoskins, 2001: Simple initial value problems and mechanisms for baroclinic growth. J. Atmos. Sci., 58, 38-49.

Barkmeijer, J., 1996: Constructing fast-growing perturbations for the nonlinear regime. J. Atmos. Sci., 53, 2838-2851.

Bretherton, F. P., 1966: Critical layer instability in a baroclinic flow. Quart. J. Roy. Meteor. Soc., 92, 325-334.

Buizza, R., and T. N. Palmer, 1995: The singular-vector structure of the atmospheric global circulation. J. Atmos. Sci., 52, 1434 1456.

Cehelsky, P., and K. K. Tung, 1991: Nonlinear baroclinic adjustment. J. Atmos. Sci., 48, 1930-1947.

Charney, J. G., 1947: The dynamics of long waves in a baroclinic westerly current. J. Meteor., 4, 135-162.

— jets in a rotating atmosphere. J. Atmos. Sci., 19, 159-172.

Duan, W. S., M. Mu, and B. Wang, 2004: Conditional nonlinear optimal perturbations as the optimal precursors for El NiñoSouthern Oscillation events. J. Geophys. Res., 109, D23105, doi:10.1029/2004JD004756.

Eady, E. T., 1949: Long waves and cyclone waves. Tellus, 1, 33-52.

Edmon, H. J., B. J. Hoskins, and M. E. McIntyre, 1980: EliassenPalm cross sections for the troposphere. J. Atmos. Sci., 37, 2600-2616.

Farrell, B. F., 1982: The initial growth of disturbances in baroclinic flows. J. Atmos. Sci., 39, 1663-1686.

_ , and P. J. Ioannou, 1996: Generalized stability theory. Part I: Autonomous operators. J. Atmos. Sci., 53, 2025-2040.

Gilmour, I., L. A. Smith, and R. Buizza, 2001: Linear regime du- ration: Is 24 hours a long time in synoptic weather forecasting? J. Atmos. Sci., 58, 3529-3539.

Gutowski, W. J., Jr., 1985: Baroclinic adjustment and midlatitude temperature profiles. J. Atmos. Sci., 42, 1733-1745.

Hart, J. E., 1981: Wavenumber selection in nonlinear baroclinic instability. J. Atmos. Sci., 38, 400-408.

Heifetz, E., and J. Methven, 2005: Relating optimal growth to counterpropagating Rossby waves in shear instability. Phys. Fluids, 17, 064 107, doi:10.1063/1.1937064.

, C. H. Bishop, B. J. Hoskins, and J. Methven, 2004: The counter-propagating Rossby-wave perspective on baroclinic instability. I: Mathematical basis. Quart. J. Roy. Meteor. Soc., 130, 211-231.

Hoskins, B. J., and M. M. Coutinho, 2005: Moist singular vectors and the predictability of some high impact European cyclones. Quart. J. Roy. Meteor. Soc., 131, 581-601.

- R. Buizza, and J. Badger, 2000: The nature of singular vector growth and structure. Quart. J. Roy. Meteor. Soc., 126, 15651580.

Joly, A., 1995: The stability of steady fronts and the adjoint method: Nonmodal frontal waves. J. Atmos. Sci., 52, 30823108.

Kim, H. M., and M. C. Morgan, 2002: Dependence of singular vector structure and evolution on the choice of norm. J. Atmos. Sci., 59, 3099-3116.

Lacarra, J.-F., and O. Talagrand, 1988: Short-range evolution of small perturbations in a barotropic model. Tellus, 40A, 81-95.

Lapeyre, G., and I. M. Held, 2004: The role of moisture in the dynamics and energetics of turbulent baroclinic eddies. J. Atmos. Sci., 61, 1693-1710.

$\mathrm{Mu}$, M., 2000: Nonlinear singular vectors and nonlinear singular values. Sci. China, 43, 375-383.

- , and Z. Zhang, 2006: Conditional nonlinear optimal perturbations of a two-dimensional quasigeostrophic model. J. Atmos. Sci., 63, 1587-1604.

_ W. W. Duan, and B. Wang, 2003: Conditional nonlinear optimal perturbation and its applications. Nonlinear Processes Geophys., 10, 493-501.

— L. Sun, and H. A. Dijikstra, 2004: The sensitivity and stability of the ocean's thermohaline circulation to finite amplitude perturbations. J. Phys. Oceanogr., 34, 2305-2315.

Nakamura, N., 1999: Baroclinic-barotropic adjustments in a meridionally wide domain. J. Atmos. Sci., 56, 2246-2260.

Palmer, T. N., R. Gelaro, J. Barkmeijer, and R. Buizza, 1998: Singular vectors, metrics, and adaptive observations. J. Atmos. Sci., 55, 633-653.

Pedlosky, J., 1964: An initial value problem in the theory of baroclinic instability. Tellus, 16, 12-17.

, 1979: Finite-amplitude baroclinic waves in a continuous model of the atmosphere. J. Atmos. Sci., 36, 1908-1924.

_ 1987: Geophysical Fluid Dynamics. 2nd ed. Springer-Verlag, $710 \mathrm{pp}$.

_ , and P. Klein, 1991: The nonlinear dynamics of slightly supercritical baroclinic jets. J. Atmos. Sci., 48, 1276-1286.

Phillips, N. A., 1954: Energy transformations and meridional circulations associated with simple baroclinic waves in a twolevel quasi-geostrophic model. Tellus, 6, 273-286.

Reynolds, C. A., and T. E. Rosmond, 2003: Nonlinear growth of singular-vector-based perturbations. Quart. J. Roy. Meteor. Soc., 129, 3059-3078.

Rivière, G., B. L. Hua, and P. Klein, 2001: Influence of the $\beta$ effect on nonmodal baroclinic instability. Quart. J. Roy. Meteor. Soc., 127, 1375-1388. 
Shepherd, T. G., 1983: Mean motions induced by baroclinic instability in a jet. Geophys. Astrophys. Fluid Dyn., 27, 35-72.

Smith, K. S., and G. K. Vallis, 2002: The scales and equilibration of midocean eddies: Forced-dissipative flow. J. Phys. Oceanogr., 32, 1699-1721.

Snyder, C., 1999: Error growth in flows with finite-amplitude waves or coherent structures. J. Atmos. Sci., 56, 500-506.

, and A. Joly, 1998: Development of perturbations within growing baroclinic waves. Quart. J. Roy. Meteor. Soc., 124, 1961-1983.
Toth, Z., and E. Kalnay, 1993: Ensemble forecasting at NMC: The generation of perturbations. Bull. Amer. Meteor. Soc., 74, 2317-2330.

Wächter, A., and L. T. Biegler, 2006: On the implementation of a primal-dual interior point filter line search algorithm for large-scale nonlinear programming. Math. Program., 106, $25-$ 57.

Zou, X., I. M. Navon, and J. Sela, 1993: Variational data assimilation with moist threshold processes using the NMC spectral model. Tellus, 45A, 370-387. 